\title{
Lower Concentrations of Glucose or Insulin Decrease the Risk of Various Types of Cancer in the Long-Lived Ames Dwarf Mouse by Increasing the Expression of p27Kip1, a Cell-Cycle Repressor Protein
}

\author{
Isao Eto \\ Department of Nutrition Sciences (DNS) and Nutrition Obesity Research Center (NORC), University of Alabama at Birmingham, \\ Birmingham, AL, USA \\ Email: etoi@uab.edu
}

How to cite this paper: Eto, I. (2020) Lower Concentrations of Glucose or Insulin Decrease the Risk of Various Types of Cancer in the Long-Lived Ames Dwarf Mouse by Increasing the Expression of p27Kip1, a Cell-Cycle Repressor Protein. American Journal of Molecular Biology, 10, 148-164.

https://doi.org/10.4236/ajmb.2020.103011

Received: March 28, 2020

Accepted: June 20, 2020

Published: June 23, 2020

Copyright $\odot 2020$ by author(s) and Scientific Research Publishing Inc. This work is licensed under the Creative Commons Attribution International License (CC BY 4.0).

http://creativecommons.org/licenses/by/4.0/ (c) (i) Open Access

\begin{abstract}
Introduction. The molecular biological mechanism of the increased incidence of the various types of cancer in obesity or type 2 diabetes in rodents or humans has largely been resolved in recent years. By contrast, the molecular biological mechanism of the decreased, not increased, incidence of the various types of cancer in the homozygous long-lived Ames dwarf mice still remains unresolved. Objective. The first objective of the present study was to investigate whether the decrease in the incidence of cancer in the homozygous long-lived Ames dwarf mice is due to the increase, not decrease, in the expression of p27Kip1, a cell cycle repressor protein. The second objective was to investigate whether the decrease in the incidence of cancer in the homozygous long-lived Ames dwarf mice is due to the decrease, not increase, in the levels of glucose or insulin. Methods. To achieve these objectives, we first performed western immunoblot analysis of the hepatic expression of p27Kip1 protein. We then performed, using a human breast cancer cell line in vitro, the luciferase reporter plasmid assay to determine whether the translation initiation activity of the p27Kip1 mRNA is increased when the concentrations of either glucose or insulin are decreased. Results and Conclusion. The results of the first objective indicated that the hepatic expression of p27Kip1 protein was up-regulated in the homozygous long-lived Ames dwarf mice as expected. We also found that the lower concentrations of glucose or insulin increased the translation initiation activity of the p27Kip1 mRNA.
\end{abstract}

\section{Keywords}

Cancer, Glucose, Insulin, Caloric Restriction, Long-Lived Ames Dwarf Mouse, p27Kip1, Cell-Cycle Repressor Protein 


\section{Introduction}

The long-lived Ames dwarf mouse is homozygous for a recessive mutation at a gene named Prophet of Pit-1 (Prop-1), which causes developmental arrest in the anterior pituitary gland [1]. These mice have physiological characteristics that are similar to the effects of caloric restriction [2] [3]. In 2003, it was reported that these mice have a delayed occurrence of presumably fatal neoplastic disease compared with their normal siblings [4]. In addition, the incidence of presumably fatal adenocarcinomas in the lung was reported to be significantly lower in the long-lived Ames dwarf mice than for their normal siblings [4] [5]. These observations about the long-lived Ames dwarf mice were made based on the decreased occurrence of the spontaneous tumors [4] [5].

The study described below regarding the long-lived Ames dwarf mice was originated from our general attempts to re-examine the controversies surrounding the association of genetic rodent models of obesity and cancer from a fresh perspective of the regulation of the expression of p27Kip1, a cell cycle repressor protein, in the tumor-free rodents. Our preliminary studies, published recently, indicated that the hepatic expression of p27Kip1 was down-regulated in the genetic rodent models of obesity [6] [7] [8]. Furthermore, we reported that the expression of p27Kip1 in the peripheral blood mononuclear cells was also down-regulated in the obese or type 2 diabetic human individuals [6] [7] [8] [9]. In contrast to these results, we now report that the expression of p27Kip1 is up-regulated, not down-regulated, in the livers of the tumor-free long-lived Ames dwarf mice [8].

The p27Kip1 is a cell cycle repressor protein that is expressed variably but ubiquitously in various tissues of the rats, mice or humans. It is a member of the family of cyclin-dependent kinase (CDK) inhibitors (CDKIs) [10]. When down-regulated, p27Kip1 up-regulates the activities of certain cyclin/CDK complexes, thereby increasing the cell cycle progression from G1 to $S$ phase, DNA replication, and the risk of developing cancer. Conversely, when up-regulated, p27Kip1 down-regulates the activities of certain cyclin/CDK complexes, thereby decreasing the cell cycle progression from G1 to S phase, DNA replication, and the risk of developing cancer. Thus, p27Kip1 could act as either a pro- or anti-cancer promotion protein, provided that the target cells have either already been initiated or are being initiated by carcinogens.

With regard to the risk of developing cancer, p27Kip1 exhibits a set of unique characteristics that are not seen in any other G1-to-S phase cell cycle regulatory proteins [11] [12] [13]. First, a relatively large number of nutritional and chemopreventive anti-cancer agents specifically increase the expression of p27Kip1 without directly affecting the expression of any other G1-to-S phase cell cycle regulatory proteins including INK4s, p57(Kip2), p21(Cip1Waf1), D-type cyclins, cyclin A, CDK2, CDK4 and CDK6 [11]. Second, the degree of increase in the expression of p27Kip1 in human breast adenocarcinoma cells in vitro is linearly and positively associated with the degree of inhibition of methylnitrosourea (MNU)-induced rat mammary adenocarcinoma in vivo [12]. This association, of 
course, does not exist for some anti-cancer agents, including tamoxiphen, which could not be converted to active anti-cancer metabolites in vitro. Lastly, unlike any other G1-to-S phase cell cycle regulatory proteins, expression of p27Kip1 is regulated primarily at the level of translation, not at the level of transcription [11]-[17]. For example, the level of expression of p27Kip1 "protein" oscillates significantly during the cell cycle, but the expression of p27Kip1 "mRNA" does not. It has been proposed by various researchers that the expression of $\mathrm{p} 27 \mathrm{Kip} 1$ could be regulated by various post-translational mechanisms, including ubiquitin-proteasome-induced degradation [18] [19] [20] [21], complex association [22], subcellular localization [22]-[27], and protein phosphorylation [27] [28] [29].

As described below in the Results section, this new approach has enabled us to largely resolve the controversies regarding the energy or insulin metabolism and the propensity of cancers. We obtained the observation that the risk of various types of cancer is increased in obesity or type 2 diabetes by decreasing the expression of p27Kip1 as a result of higher levels of glucose or insulin, whereas it is decreased in the long-lived Ames dwarf mice by increasing the expression of p27Kip1 as a result of lower levels of glucose or insulin. We believe that this study has a translational potential.

\section{Materials and Methods}

\subsection{Long-Lived Ames Dwarf Mouse and Normal Ames Control Mouse In Vivo}

\subsubsection{Animals}

Homozygous long-lived Ames dwarf mice (males) (J181312q Prop1-/-) and wild-type normal Ames control mice (males) (J181312q Prop1+/+) were purchased initially from the Jackson Laboratory (Bar Harbor, MI, USA) at 9 to 10 weeks of age, but later they were kindly and generously provided by Dr. Andrzej Bartke at the Southern Illinois University (SIU) School of Medicine (Springfield, IL, USA).

At our institution (University of Alabama at Birmingham, Birmingham, Al., USA), all procedures involving animals were reviewed and approved by the Institutional Animal Care and Use Committee (IACUC). All animals were housed in regular mouse cages on 12-h light/dark cycle (lights on at 7:00 am) and had unlimited access to food and tap water at our institution. All mice were fed Lab Diet ${ }^{\circledR} 5 \mathrm{~K} 52$, which contained approximately $19 \%$ protein and included ground wheat, dehulled soybean meal, and fish meal. All animals were sacrificed at 12 weeks of age after overnight fast and the serum and liver samples were collected from each animal, snap-frozen in liquid nitrogen, and stored at $-80^{\circ} \mathrm{C}$ until processed further.

\subsubsection{Western Immunoblot Analysis of the Hepatic Expression of p27Kip1}

The following primary antibodies against p27Kip1 were purchased from the Cell 
Signaling Technology (Danvers, MA, USA) and the Santa Cruz Biotechnology (Santa Cruz, CA, USA). Additionally, primary antibodies against GAPDH were purchased from the Santa Cruz Biotechnology.

Western immunoblot analysis of the expression of p27Kip1 was performed using the livers of the mice. The soluble protein extracts of the livers were prepared by sonicating in RIPA Lysis Buffer (Santa Cruz Biotechnology, Santa Cruz, CA, USA) containing PMSF, sodium orthovanadate and protease inhibitor cocktail and supplemented with $50 \mathrm{mmol} / \mathrm{L} \mathrm{NaF}$. The soluble protein extracts were collected by centrifugation and stored at $-80^{\circ} \mathrm{C}$ until processed for western immunoblot analysis.

For western immunoblot analysis, the soluble protein extracts (35 to $50 \mu \mathrm{g}$ protein per lane) were applied to the SDS-PAGE and, after fractionation, proteins were transferred to the nitrocellulose membrane, which was then blocked and incubated in a solution containing first primary antibody. After shaking overnight at $4^{\circ} \mathrm{C}$, the target proteins bound to the first primary antibody against p27Kip1 were further incubated in a solution containing alkaline phosphatase (AP)-conjugated secondary anti-immunoglobulin antibody and detected by chemiluminescence using TROPIX Western-Star Kit (Applied Biosystems, Foster City, CA, USA). After exposure to X-ray film, the blots were stripped using Western Re-Probe Solution (G-Biosciences, St. Louis, MO, USA), checked for removal of the chemiluminescence and then re-probed with second primary antibody against GAPDH.

Densitometric measurements of the intensity of the bands on the X-ray films were performed using UN-SCAN-IT Gel \& Graph Digitizing Software (Silk Scientific, Orem, UT, USA). Background corrections were done by four corner interpolation method and optical density calculations were performed using linear standard reflective scan method.

\subsubsection{Analysis of the Serum Levels of Glucose and Insulin}

Serum levels of glucose and insulin were determined at our laboratory (Department of Nutrition Sciences, University of Alabama at Birmingham (UAB)) (Birmingham, AL, USA). This laboratory has been jointly operated by the NIH-funded Pittman General Clinical Research Center and Nutrition Obesity Research Center at $\mathrm{UAB}$.

Glucose was determined by glucose oxidase method. Insulin was determined by double-antibody radioimmunoassay procedure using RIA Linco kits manufactured by the Linco Research (St. Charles, MO, USA).

\subsection{Human Breast Cancer Cell Line MDA-MB-231 In Vitro}

\subsubsection{MDA-MB-231 Cell Line In Vitro}

Human breast cancer cell line MDA-MB-231 in vitro was obtained from the American Type Culture Collection (Rockville, MD, USA). The cells were grown in Dulbecco's Modified Eagle's Medium (DMEM) containing $4.5 \mathrm{~g} / \mathrm{L}$ of $\mathrm{d}-(+)$-glucose, supplemented with $10 \%$ heat-inactivated FBS, $2 \%$ l-glutamine, 
and antibiotics. All cells were subcultured after trypsinization with $0.05 \%$ trypsin- $0.02 \%$ EDTA solution. The incubation was carried out at $37^{\circ} \mathrm{C}$ in a $5 \% \mathrm{CO}_{2}$ humidified chamber. The cultures were always maintained below confluency. The cells were checked periodically for mycoplasmal infection by DNA fluorochrome staining.

\subsubsection{Other Materials Used for the Study of MDA-MB-213 Cell Line In Vitro}

LY 294002, rapamycin, d-(+)-glucose, and rotenone were obtained from Sigma-Aldrich (St. Louis, MO, USA). Triciribine, AG1024, AGL2263, IGF-1R inhibitor PPP, compound $C$, and metformin were obtained from Calbiochem/EMD (San Diego, CA, USA). AICA riboside was purchased from Phoenix Pharmaceuticals, Inc. (Belmont, CA, USA). Dulbecco's Modified Eagle's Medium (DMEM) labeling kit was obtained from Chemicon International (Temecula, CA, USA).

\subsubsection{Luciferase Reporter Plasmid Used to Estimate the Translation Initiation Activity of the p27Kip1 mRNA Corresponding to the 5'-Upstream Untranslated Region (5'UTR) of the p27Kip1 Gene}

Luciferase reporter plasmid containing from -575 to -1 untranslated region (p27Kip1-5'UTR) of the p27Kip1 mRNA [6] [7] in the proximal 5'-upstream region of the p27Kip1 gene was used to transfect the cells. The control luciferase reporter plasmid that does not contain the insert was also prepared to test if any unknown pro-cancer agents or nutritional/chemopreventive anti-cancer agents were exerting any spurious effects on the backbone, rather than the insert, of the luciferase reporter plasmid. We found that none of the agents tested exerted any spurious effects on the backbone of the luciferase reporter plasmid in the human breast cancer cells used.

\subsubsection{Transfection and Luciferase Reporter Assay}

Transfections were performed according to the published protocol [30] using FuGENE 6 purchased from the Roche Applied Science (Indianapolis, IN, USA). In brief, 24 hours before reporter transfection, the cells were seeded into a $60-\mathrm{mm}$ tissue culture dish at a density of $1.5 \times 10^{5}$ cells/dish and incubated at $37^{\circ} \mathrm{C}$ in a $5 \% \mathrm{CO}_{2}$ humidified chamber. Reporter transfection was then carried out with $1 \mu \mathrm{g}$ of luciferase reporter plasmid and $0.2 \mu \mathrm{g}$ of $\mathrm{pSV}$ - $\beta$-galactosidase internal control plasmid (Promega, Madison, WI, USA) mixed with $3 \mu \mathrm{L}$ of FuGENE 6 solution in $3 \mathrm{~mL}$ of FBS-free DMEM supplemented with only 2\% l-glutamine. A minimum of 5-hour incubation at $37^{\circ} \mathrm{C}$ was needed for transient transfection, followed by 18 -hour incubation at $37^{\circ} \mathrm{C}$ in a $5 \% \mathrm{CO}_{2}$ humidified chamber in normal DMEM with $10 \%$ FBS for recovery. The transfected cells were then starved in DMEM with $0.2 \% \mathrm{FBS}$ at $37^{\circ} \mathrm{C}$ in a $5 \% \mathrm{CO}_{2}$ humidified chamber for 24 hours.

The resulting cells were treated with either various compounds or vehicle controls in DMEM with $0.2 \% \mathrm{FBS}$ at $37^{\circ} \mathrm{C}$ in a $5 \% \mathrm{CO}_{2}$ humidified chamber for 24 hours. For studies with zero concentration of glucose, DMEM labeling kit was 
purchased from the Chemicon International (Temecula, CA, USA), but only glucose was omitted from the kit. The cells, transfected with luciferase reporter plasmid in normal DMEM with 10\% FBS and starved in normal DMEM with $0.2 \%$ FBS for 24 hours as described above, were then incubated in either normal concentration $(5.55 \mathrm{mM})$ or zero concentration of $\mathrm{d}-(+)$-glucose in glucose-deficient DMEM with $0.2 \% \mathrm{FBS}$ at $37^{\circ} \mathrm{C}$ in a $5 \% \mathrm{CO}_{2}$ humidified chamber for 24 hours.

After 24 hours, the treated cells were collected and lysed using Reporter Lysis Buffer (Promega, Madison, WI). The resulting cell lysates were assayed for luciferase activity using Luciferase Assay Kit (Promega, Madison, WI, USA) and TD-20/20 Luminometer (Turner Designs, Sunnyvale, CA, USA). $\beta$-Galactosidase activity was measured using chlorophenol red- $\beta$-d-galactopyranoside (CPRG) (Sigma-Aldrich, St. Louis, MO, USA) as a substrate.

\subsection{Statistical Analysis}

An experimental value with statistical significance of $\mathrm{P} \leq 0.05$ compared to the control by Student's $t$ test was indicated as a single asterisk on top of the vertical bar.

\section{Results}

\subsection{Animal Studies Using Long-Lived Ames Dwarf Mouse and Normal Ames Control Mouse In Vivo}

\subsubsection{The Levels of the Hepatic Expression of p27Kip1, a Cell Cycle Repressor Protein, Were Significantly Higher in the Long-Lived Ames Dwarf Mouse Relative to the Normal Ames Control Mouse (Figures 1(a)-(d))}

To assess the regulation of the hepatic expression of p27Kip1, a cell cycle repressor protein, the long-lived Ames dwarf mice and normal Ames control mice were used for this study (Figure 1(a)). All mice were obtained at 9 to 10 weeks of age and sacrificed at 12 weeks of age. These mice were all males because the levels of the expression of p27Kip1 were generally gender neutral based on our preliminary studies. These animals were also considered "tumor-free" based on the following two criteria: 1) they were generally tumor free at this younger age as confirmed by our resident pathologist and the commercial suppliers and also 2) they had not been treated with any known chemical carcinogens.

The body weights of the long-lived Ames dwarf mice were significantly lower-by about 63\%-relative to the normal Ames control mice (Figure 1(b)). At 12 weeks of age, the average body weight of the long-lived Ames dwarf mice was about 10 grams and that of the normal Ames control mice was about 27 grams. The P-values in the difference in the body weights between long-lived Ames dwarf mice and normal Ames control mice were below 0.01 . This may be due to the fact that the homozygous dwarf mice were not fertile and, therefore, dwarf gene must be maintained in the heterozygotes. So, it is likely that the homozygous long-lived Ames dwarf mice and the wild-type normal Ames control mice 
were siblings.

In contrast to the body weights, western immunoblot analyses of the levels of the hepatic expression of p27Kip1 were significantly higher-by about 74\%-in the long-lived Ames dwarf mice relative to those of the normal Ames control mice (Figure 1(c)).

Figure 1(d) shows the overview of the molecular biological effects of the increasing expression of p27Kip1, a cell cycle repressor protein, on the cell cycle in the long-lived Ames dwarf mice relative to the normal Ames control mice. The results of the preliminary studies have indicated that the increased expressions of p27Kip1 decrease the risk of cancer by inhibiting the G1-to-S phase transition of the cell cycle, thereby decreasing the DNA replication in the $\mathrm{S}$ phase and cell division in the $\mathrm{M}$ phase. Numerous nutritional and chemopreventive anti-cancer agents decrease the risk of cancer by increasing the expression of p27Kip1. These effects of the increased expressions of p27Kip1 appear to be specific to the p27Kip1. No other cell cycle regulatory proteins are known to cause these effects.

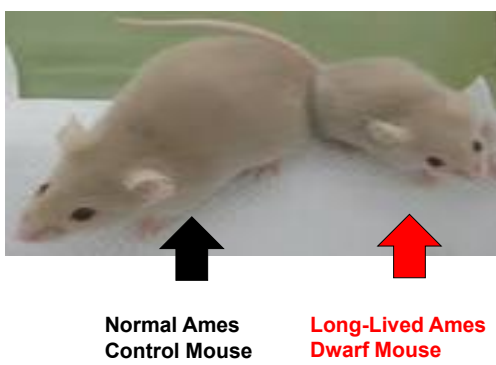

(a)

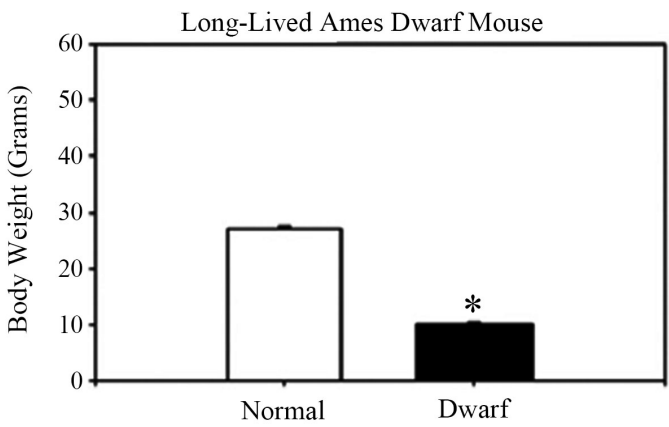

(b)

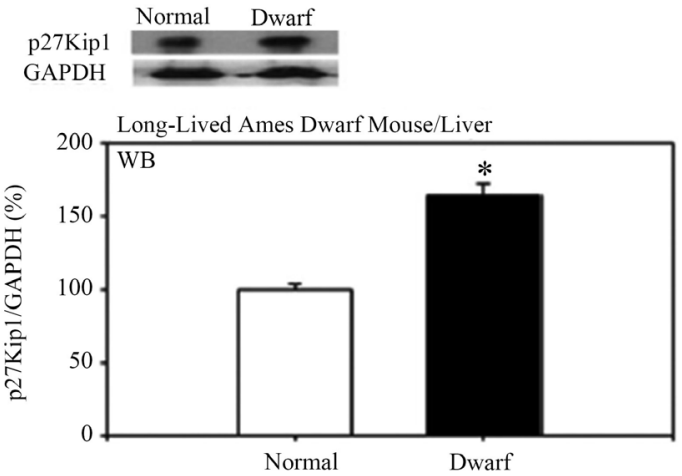

(c) 


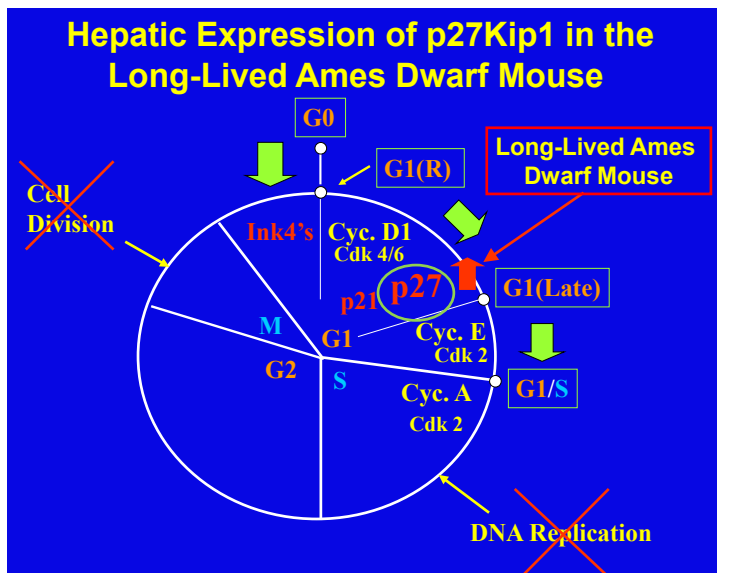

(d)

Figure 1. Hepatic expression of p27Kip1, a cell cycle repressor protein, was significantly higher in the long-lived Ames dwarf mouse relative to the normal Ames control mouse. (a) shows the images of the long-lived Ames dwarf mouse on the right side and the normal Ames control mouse on the left side. (b) shows the body weights of the three long-lived Ames dwarf mice on the right side and the three normal control mice on the left side. (c) shows the western immunoblot analysis of the hepatic expression of p27Kip1, a cell cycle repressor protein, in the three long-lived Ames dwarf mice on the right side and the three normal Ames control mice on the left side. All mice were sacrificed at 12 weeks of age and all were males because the expression of p27Kip1 was gender neutral based on our preliminary studies. (d) shows the overview of the effects of increasing the expression of p27Kip1, a cell cycle repressor protein, on the cell cycle in the long-lived Ames dwarf mouse.

\subsubsection{Serum Levels of Glucose and Insulin Were Significantly Lower in the Long-Lived Ames Dwarf Mouse Relative to the Normal Ames Control Mouse (Figures 2(a)-(c))}

The serum concentrations of glucose were significantly lower-by about $68 \%$-relative to the normal Ames control mice (Figure 2(a)). At 12 weeks of age, the average concentrations of the serum glucose of the long-lived Ames dwarf mice were about $112 \mathrm{mg} / \mathrm{dl}$ and those of the normal Ames control mice were about $350 \mathrm{mg} / \mathrm{dl}$. Similarly, the serum concentrations of insulin were also significantly lower-by about 78\%-relative to the normal Ames control mice (Figure 2(b)). At 12 weeks of age, the average concentrations of the serum insulin of the long-lived Ames dwarf mice were about $0.125 \mathrm{ng} / \mathrm{ml}$ and those of the normal Ames control mice were about $0.58 \mathrm{ng} / \mathrm{ml}$.

Figure 2(c) illustrates the tentative hypothesis of how the lower concentrations of serum glucose or insulin might inhibit the cell cycle by increasing the expression of p27Kip1, a cell cycle repressor protein, in the long-lived Ames dwarf mouse. This hypothesis is identical to that presented above in Figure 1(d) except that we now propose more specifically that the lower concentrations of serum glucose or insulin are the ones that might be responsible for increasing the expression of p27Kip1, a cell cycle repressor protein, thereby decreasing the risk of various types of cancer in the long-lived Ames dwarf mouse. 


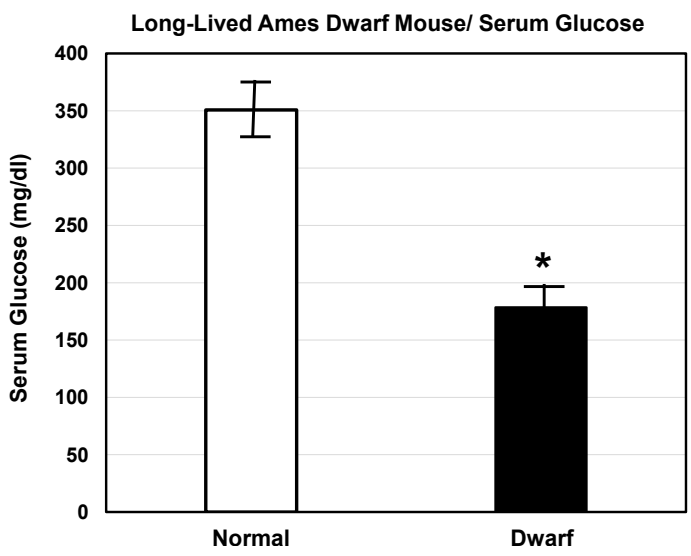

(a)

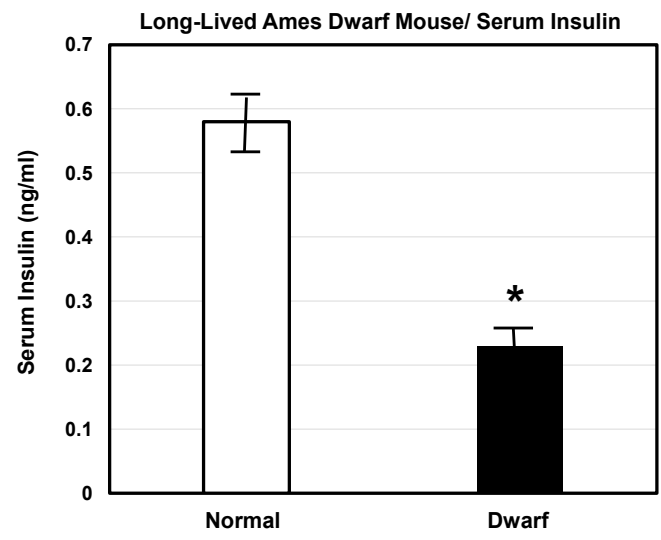

(b)

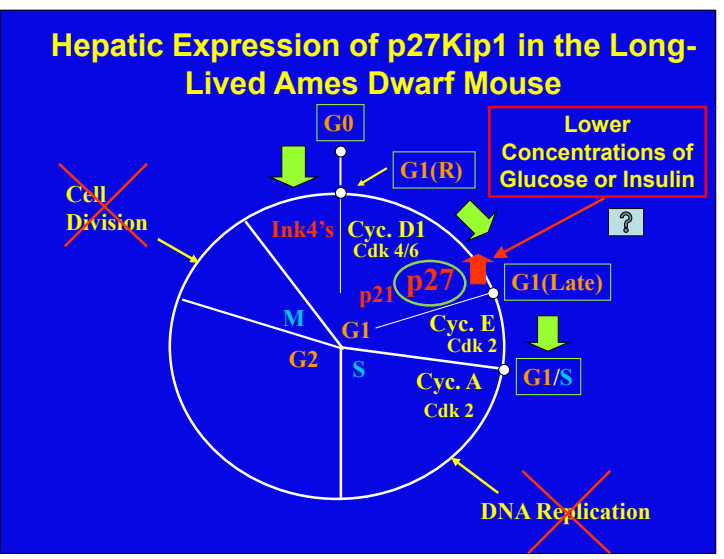

(c)

Figure 2. Serum levels of glucose and insulin were significantly lower in the long-lived Ames dwarf mouse relative to the normal Ames control mouse. (a) shows that the levels of the serum glucose were significantly lower in the three long-lived Ames dwarf mice on the right side relative to the three normal Ames control mice on the left side. Similarly, (b) shows that the levels of the insulin were also significantly lower in the three long-lived Ames dwarf mice on the right side relative to the three normal Ames control mice on the left. The mice used in these studies were all males at 12 weeks of age. (c) illustrates the hypothesis of how the lower serum levels of glucose or insulin might inhibit the cell cycle by increasing the expression of p27Kip1, a cell cycle repressor protein, in the long-lived Ames dwarf mouse. 
3.2. Cell Culture Studies to Investigate the Effects of the Lower Concentrations of Glucose or Insulin on the Expression of p27Kip1 Using Human Breast Cancer Cell Line MDA-MB-231 In Vitro

\subsubsection{Primary Structure of the Luciferase Reporter Plasmid Used to} Estimate the Translation Initiation Activity of the p27Kip1 mRNA That Corresponds to the 5'-Upstream Untranslated Region (5'UTR) of the p27Kip1 mRNA (Figure 3)

Molecular biological mechanism of the regulation of the expression of p27Kip1 protein is unusual. First of all, expression of p27Kip1 is regulated primarily at the level of translation, not at the level of transcription. Second, its mode of translation initiation is cap independent, not cap dependent. Thus, investigation of the potential regulation of the translation initiation activity of the p27Kip1 mRNA by lower concentrations of glucose or insulin must be performed carefully.

Our preliminary studies have indicated that the best approach to estimate the translation initiation activity of the p27Kip1 mRNA by glucose or insulin was to use the luciferase reporter plasmid that contains only a portion of the p27Kip1 gene that corresponds to the proximal 5'-upstream untranslated region (5'UTR) of the p27Kip1 mRNA. The primary RNA structure of this luciferase reporter plasmid is reproduced in Figure 3. It consists of the RNA bases from -575 to -1 . A more comprehensive description of this portion of the p27Kip1 mRNA will be presented later in the Discussion section of this manuscript. We reported recently that the molecular downstream pathways of how higher concentrations of glucose or insulin decrease the translation initiation activity of p27Kip1 mRNA by transiently transfecting this luciferase reporter plasmid into the MDA-MB-231 human breast cancer cells in vitro [6] [7]. It made sense, therefore, to use the same approach to investigate the opposite situation of how lower concentrations of glucose or insulin decrease the risk of various types of cancer by increasing the translation initiation activity of p27Kip1 mRNA.

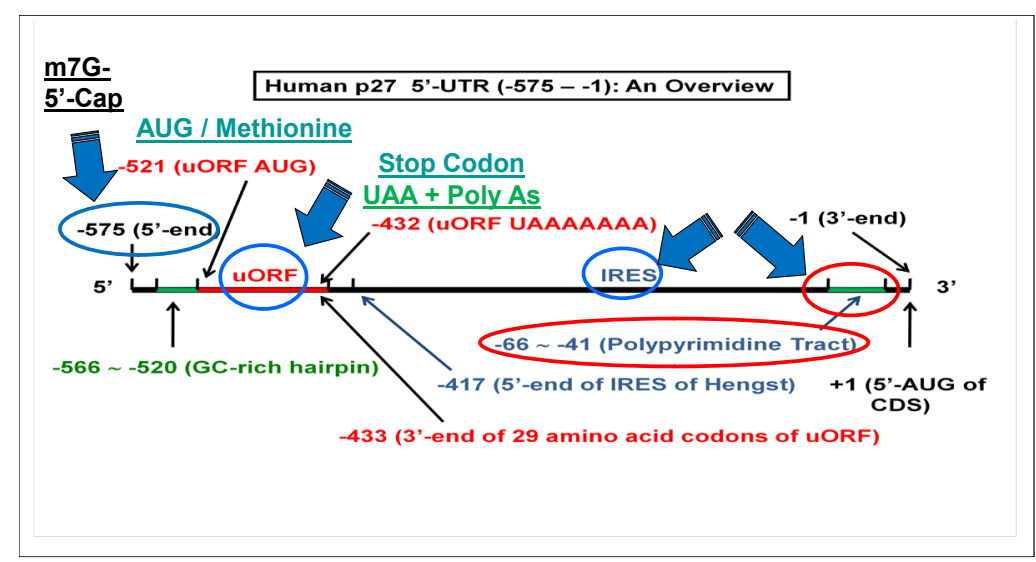

Figure 3. Primary structure of the upstream 5'-untranslated region of p27Kip1 mRNA. It consists of the bases from -1 to -575 . A more comprehensive description of this portion of the p27Kip1 mRNA will be presented in the Discussion section of this manuscript. 
3.2.2. Lower Concentrations of D-(+)-Glucose Increased the Translation Initiation Activity of p27Kip1 mRNA in MDA-MB-231 Cells In Vitro (Figure 4(a) and Figure 4(b))

The results shown in Figure 4(a) indicate that the removal of d-(+)-glucose from the cell culture medium for 24 hours significantly up-regulated the translation initiation activity of p27Kip1 mRNA (p27Kip1-5'UTR) relative to the normal concentration $(5.55 \mathrm{mM})$ of glucose. The significant up-regulation of the translation initiation activity of p27Kip1 mRNA was also observed in the lower concentration $(5.55 \mathrm{mM})$ of glucose relative to that when higher concentration $(20 \mathrm{mM})$ of glucose was added to the culture medium for 24 hours.

Figure 4(b) shows that those compounds, circled in orange color, increased the translation initiation activities of p27Kip1 mRNA in the MDA-MB-231 cells in vitro. These compounds included rotenone, AICA riboside, metformin, and rapamycin. The other compounds, circles in green color, decreased the translation initiation activities of p27Kip1 mRNA in the same cells in vitro. These compounds included compound $\mathrm{C}$ and one or more of the branched chain amino acids. The 4E-BP1 (eukaryotic translation initiation factor 4E-binding protein 1 (eIF4E-BP)), circled in red color, decreased the protection of 5 '-end cap of the p27Kip1 mRNA (p27Kip1-5'UTR), thereby increasing the cap-independent translation initiation activity of the p27Kip1 mRNA.

\subsubsection{Lower Concentrations of Insulin Also Increased the Translation Initiation Activity of the p27Kip1 mRNA in MDA-MB-231 Cells In Vitro (Figure 5(a) and Figure 5(b))}

Figure 5(a) shows that certain inhibitors of insulin receptor appeared to have significantly and specifically increased the translation initiation activity of p27Kip1 mRNA (p27Kip1-5'UTR) in these cells in vitro. Of the three inhibitors used, two of them-IGF-IR inhibitor PPP and AGL2263 (IR and IGF-1R inhibitor) - significantly and specifically increased the translation initiation activity of p27Kip1 mRNA (p27Kip1-5'UTR) in MDA-MB-231 cells in vitro, but AG1024 (IGF-1R inhibitor) failed to increase it. These results suggested that the lower concentrations of insulin increased the translation initiation activity of p27Kip1 mRNA in vitro.

Figure 5(b) shows that those compounds, circled in orange color, increased the translation initiation activities of p27Kip1 mRNA in the MDA-MB-231 cells in vitro. These compounds included 1 ) the inhibitor of the receptor tyrosine kinase for the platelet derived growth factor; 2) LY 294,002 (inhibitor of phosphoinositide 3-kinase); and 3) triciribine (inhibitor of Akt/PKB).

The downstream pathway of increasing the translational activity of p27Kip1 mRNA by lower concentrations of insulin merges with the downstream pathway of lowering the concentrations of $\mathrm{d}-(+)$-glucose at the point of the tuberous sclerosis proteins, Subsequently, the $4 \mathrm{E}-\mathrm{BP} 1$, that is circled in red color, acts in the same way as for $\mathrm{d}-(+)$-glucose; the $4 \mathrm{E}-\mathrm{BP} 1$ decreases the protection of 5 -end cap of the p27Kip1 mRNA (p27Kip1-5'UTR), thereby increasing the cap-independent translation initiation of the p27Kip1 mRNA. 


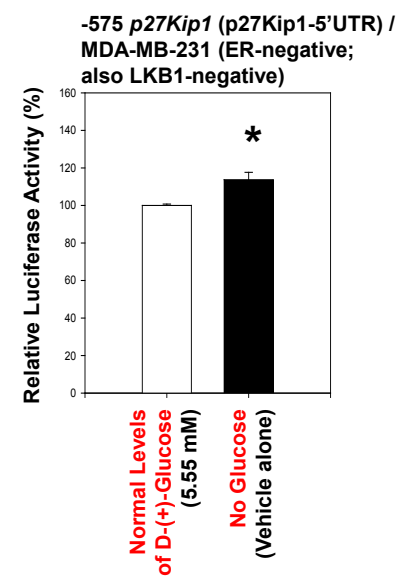

(a)

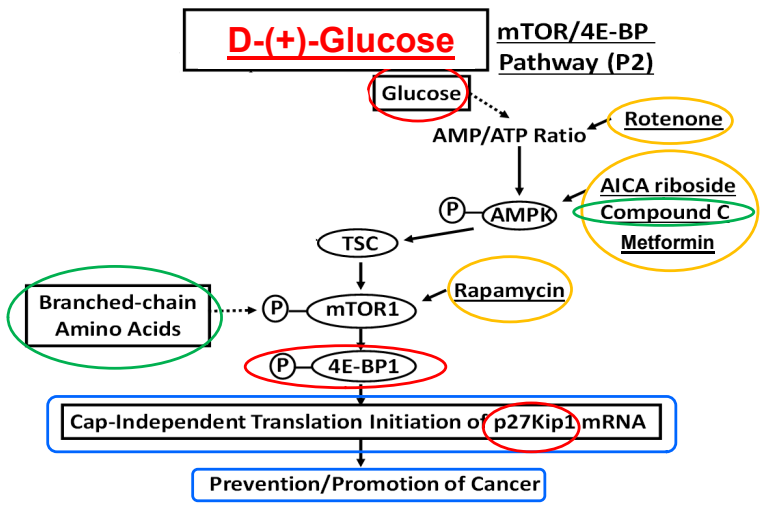

(b)

Figure 4. Lower concentrations of $\mathrm{D}-(+)$-glucose increased the translation initiation activity of p27Kip1 mRNA (p27Kip1-5'UTR) in MDA-MB-231 cells in vitro. (a) shows that the relative luciferase activity of the translation initiation of the p27Kip1 mRNA was significantly higher when no glucose was added to the culture medium for 24 hours relative to that when normal concentration $(5.55 \mathrm{mM})$ of $\mathrm{d}-(+)$-glucose was added. (b) shows the downstream pathway of how the lower concentrations of $\mathrm{D}-(+)$-glucose increased the translation initiation activity of p27Kip1 mRNA in vitro. The metabolites that increased the translation initiation activity of p27Kip1 mRNA are marked in orange circle, while those that inhibited are marked in green circle.

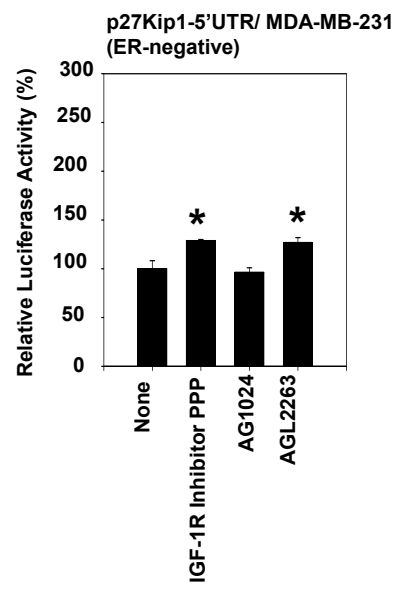

(a) 


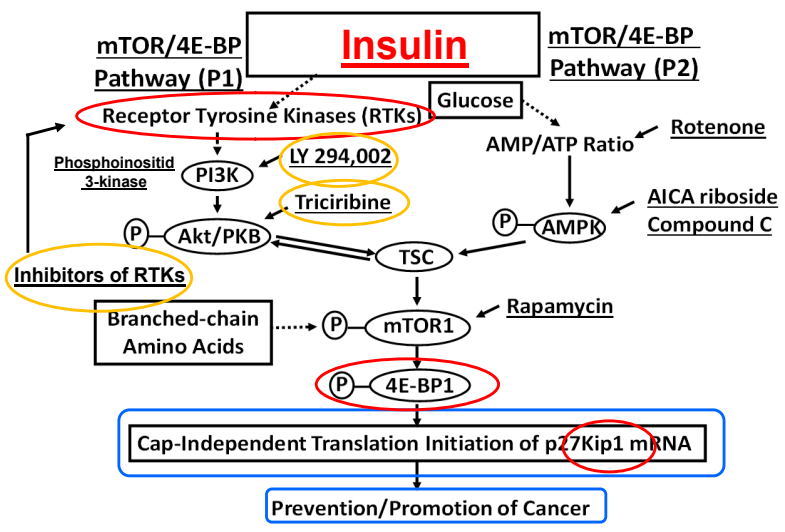

(b)

Figure 5. Lower concentrations of insulin also increased the translation initiation activity of p27Kip1 mRNA (p27Kip1-5'UTR) in the MDA-MB-231 cells in vitro. (a) shows that certain inhibitors of insulin receptor appeared to have significantly and specifically increased the translation initiation activity of p27Kip1 mRNA (p27Kip1-5'UTR) in these cells in vitro. (b) shows the downstream pathway of how the lower concentrations of insulin might have also increased the translation initiation activity of the p27Kip1 mRNA in these cells in vitro. The metabolites that increased the translation initiation activity of p27Kip1 mRNA are marked in orange circle.

\section{Discussion}

Our previous preliminary studies indicated that the increased expressions of p27Kip1, a cell cycle repressor protein, decrease the risk of cancer by inhibiting the G1-to-S phase transition of the cell cycle, thereby decreasing the DNA replication in the $\mathrm{S}$ phase and cell division in the $\mathrm{M}$ phase. Numerous nutritional and chemopreventive anti-cancer agents are now known to decrease the risk of cancer by increasing the expression of p27Kip1 protein. The effects of the increased expressions of p27Kip1 protein are specific. The cell cycle regulatory proteins other than p27Kip1 are not known to cause these effects. Additionally, the experimental results presented above indicate that the lower concentrations of glucose or insulin decrease the risk of various types of cancer in the long-lived Ames dwarf mice by increasing the expression of p27Kip1 protein. By contrast, our previous preliminary studies, published recently, indicated that higher, not lower, concentrations of glucose or insulin increase the risk of various types of cancer in obesity or type 2 diabetes by decreasing the expression of p27Kip1 protein [6] [7].

As we stated briefly above in the Results section, the molecular biological mechanism of the expression of p27Kip1 protein is unusual. First of all, expression of p27Kip1 protein is regulated primarily at the level of translation, not at the level of transcription. Second, its mode of translation initiation is cap independent, not cap dependent. Therefore, investigation of the potential regulation of the translation initiation activity of the p27Kip1 mRNA by lower concentrations of glucose or insulin needs to be performed carefully.

The primary RNA structure of the unusually long upstream 5 '-untranslated region of p27Kip1 mRNA (p27Kip1 5'-UTR) was briefly introduced, but not fully 
outlined, above in the Results section (Figure 3). The upstream 5'-uptranslated region of p27Kip1 mRNA contains RNA bases from -575 to -1 . Its primary RNA structure contains the following three sequence motifs, namely 1) 5 '-end cap at $-575,2)$ upstream open reading frame (uORF) consisting of AUG codon beginning at -521 and followed by 28 additional amino acid codons and then a stop codon plus poly A tail beginning at -433 , and 3) internal ribosome entry site (IRES) from -66 to -41 . The AUG codon of the main open reading frame of the p27Kip1 mRNA begins at +1 .

The lower concentrations of glucose decrease the associated downstream energy metabolism of glucose including the mitochondrial electron transfer chain, ATP production, AMPK and TSC (tuberous sclerosis complex) proteins, mTORC1, and eIF4E-BP1 (eukaryotic translation initiation factor 4E-binding protein 1 -also known as $4 \mathrm{E}-\mathrm{BP} 1)$. The $4 \mathrm{E}-\mathrm{BP} 1$ lowers the protection of the 5 '-end cap of the upstream 5'-untranslated region of p27Kip1 mRNA (p27Kip1 5 '-UTR), thereby activating the cap-independent translation initiation of the p27Kip 1 mRNA by diverting the 40 S ribosome to the internal ribosome entry site.

The lower concentrations of insulin also decrease the associated downstream RTKs (receptor tyrosine kinases), PI3Ks (phosphoinositide 3-kinases) and TSC (tuberous sclerosis complex) proteins. Subsequent downstream pathway of the lower concentration of insulin is identical to that of the lower concentrations of glucose; the lower concentrations of insulin activate the cap-independent translation initiation of the p27Kip1 mRNA by way of 4E-BP1.

\section{Ethical Statement}

Prior to the commencement of this study, all procedures involving rodents were reviewed and approved by the Institutional Animal Care and Use Committee (IACUC) at the University of Alabama at Birmingham (Birmingham, AL, USA).

\section{Acknowledgements}

The author is greatly indebted to Dr. Andrzej Bartke at the Southern Illinois University (SIU) School of Medicine (Springfield, IL, USA) for having kindly and generously provided us with homozygous (Prop1-/-) long-lived Ames dwarf mice and the wild-type normal Ames control mice. The author is also extremely grateful to Mrs. Maryellen Williams and Mrs. Zhaojing Cindy Zeng at the University of Alabama at Birmingham (Birmingham, AL, USA) for performing metabolite analyses of the rodent sera. Additionally, the author greatly appreciates the research environment provided by the Nutrition Obesity Research Center at the University of Alabama at Birmingham (UAB) (Birmingham, AL, USA). The content of this study, however, is solely the responsibility of the author.

\section{Conflicts of Interest}

We declare that no actual or potential conflicts of interest exist that might, in 
principle, influence our scientific judgment.

\section{References}

[1] Sornson, M.W., Wu, W., Dansen, J.S., Flynn, S.E., Norman, D.J., O’Connell, S.M., Gukovsky, I., Carriere, C., Ryan, A.K., Miller, A.P., Zuo, L., Gleiberman, A.S., Anderson, B., Beamer, W.G. and Rosenfeld, M.G. (1996) Pituitary Lineage Determination by the Prophet of Pit-1 Homeodomain Factor Defective in Ames Dwarfism. Nature, 384, 327-333. https://doi.org/10.1038/384327a0

[2] Ikeno, Y., Lew, C.M., Cortez, L.A., Webb, C.R., Lee, S. and Hubbard, G.B. (2006) Do Long-Lived Mutant and Calorie-Restricted Mice Share Common Anti-Aging Mechanisms? A Pathological Point of View. Age, 28, 163-171. https://doi.org/10.1007/s11357-006-9007-7

[3] Wiesenborn, D.S., Galvez, E.J.C., Spinel, L., Victoria, B., Allen, B., Schneider, A., Gesing, A., Al-Regaiey, K.A. Strowig, T., Schaefer, K.H. and Masternak, M.M. (2019) The Role of Ames Dwarfism and Calorie Restriction on Gut Microbiota. The Journal of Gerontology: Biological Sciences, 20, 1-8. https://doi.org/10.1093/gerona/glz236

[4] Ikeno, Y., Bronson, R.T., Hubbard, G.B., et al. (2003) Delayed Occurrence of Fatal Neoplastic Diseases in Ames Dwarf Mice: Correlation to Extended Longevity. The Journal of Gerontology Ser. A, 58, B291-B296.

https://doi.org/10.1093/gerona/58.4.B291

[5] Sharp, Z.D. and Bartke, A. (2005) Evidence for Down-Regulation of Phosphoinositide 3-Kinase/Akt/Mammalian Target of Rapamycin (PI3K/Akt/mTOR)-Dependent Translation Regulatory Signaling Pathways in Ames Dwarf Mice. The Journals of Gerontology Ser. A, 60, B293-B300. https://doi.org/10.1093/gerona/60.3.293

[6] Eto, I. (2020) Higher Concentrations of Glucose or Insulin Increase the Risk of Various Types of Cancer in Obesity or Type 2 Diabetes by Decreasing the Expression of p27Kip1, a Cell Cycle Repressor Protein. American Journal of Molecular Biology, 10, 1-11. https://doi.org/10.4236/ajmb.2020.101001

[7] Eto, I. (2018) Expression of p27Kip1, a Cell Cycle Repressor Protein with Dual Roles for Both Cancer Prevention and Promotion, Is Regulated Primarily at the Level of Unusual p27Kip1 mRNA-A Short Concept Proposal. American Journal of Molecular Biology, 8, 186-193. https://doi.org/10.4236/ajmb.2018.83016

[8] Eto, I. (2013) Expression of p27Kip1, a Cell Cycle Repressor Protein, Is Inversely Associated with Potential Carcinogenic Risk in the Genetic Rodent Models of Obesity and Long-Lived Ames Dwarf Mice. Metabolism Clinical and Experimental, 62, 873-887. https://doi.org/10.1016/j.metabol.2013.01.001

[9] Eto, I. (2014) Expression of p27(Kip1), a Cyclin-Dependent Kinase Inhibitor, in Human Peripheral Blood Mononuclear Cells Is Inversely Associated with Potential Carcinogenic Risk in Obese Type 2 Diabetic Individuals Relative to Lean Normal Controls. American Journal of Molecular Biology, 3, 113-128. https://doi.org/10.4236/ajmb.2014.43013

[10] Alkarain, A. and Slingerland, J. (2004) Deregulation of p27 by Oncogenic Signaling and Its Prognostic Significance in Breast Cancer. Breast Cancer Research, 6, 13-21. https://doi.org/10.1186/bcr722

[11] Eto, I. (2006) Nutritional and Chemopreventive Anti-Cancer Agents Up-Regulate Expression of p27Kip1, a Cyclin-Dependent Kinase Inhibitor, in Mouse JB6 Epidermal and Human MCF7, MDA-MB-321 and AU565 Breast Cancer Cells. Cancer Cell International, 6, 20. https://doi.org/10.1186/1475-2867-6-1 
[12] Eto, I. (2010) Upstream Molecular Signaling Pathways of p27(Kip1) Expression: Effects of 4-Hydroxytamoxifen, Dexamethasone, and Retinoic Acids. Cancer Cell International, 10, 3. https://doi.org/10.1186/1475-2867-10-3

[13] Eto, I. (2011) Upstream Molecular Signaling Pathways of p27(Kip1) Expression in Human Breast Cancer Cells in Vitro: Differential Effects of 4-Hydroxytamoxifen and Deficiency of Either D-(+)-glucose or L-Leucine. Cancer Cell International, 11, 31. https://doi.org/10.1186/1475-2867-11-31

[14] Hengst, L. and Reed, S.I. (1996) Translational Control of p27Kip1 Accumulation during the Cell Cycle. Science, 71, 1861-1864. https://doi.org/10.1126/science.271.5257.1861

[15] Agrawal, D., Hauser, P., McPherson, F., et al. (1996) Repression of p27(kip1) Synthesis by PDGF in balb/c 3 T3 Cells. Molecular and Cell Biology, 16, 4327-4336. https://doi.org/10.1128/MCB.16.8.4327

[16] Millard, S.S., Yan, J.S., Nguyen, H., et al. (1997) Enhanced Ribosomal Association of p27(Kip1) mRNA Is a Mechanism Contributing to Accumulation during Growth Arrest. Journal of Biological Chemistry, 19, 7093-7098. https://doi.org/10.1074/jbc.272.11.7093

[17] Goepfert, U., Kullmann, M. and Hengst, L. (2003) Cell Cycle-Dependent Translation of p27 Involves a Responsive Element in Its 5'-UTR That Overlaps with a uORF. Human Molecular Genetics, 12, 1767-1779. https://doi.org/10.1093/hmg/ddg177

[18] Pagano, M., Tam, S.W., Theodoras, A.M., et al. (1995) Role of the Ubiquitin-Proteasome Pathway in Regulating Abundance of the Cyclin-Dependent Kinase Inhibitor p27. Science, 269, 682-685. https://doi.org/10.1126/science.7624798

[19] Malek, N.P., Sundberg, H., McGrew, S., et al. (2001) A Mouse Knock-In Model Exposes Sequential Proteolytic Pathways That Regulate p27Kip1 in G1 and S Phase. Nature, 413, 323-327. https://doi.org/10.1038/35095083

[20] Hara, T., Kamura, T., Nakayama, K., et al. (2001) Degradation of p27(Kip1) at the G(0)-G(1) Transition Mediated by a Skp2-Independent Ubiquitination Pathway. Journal of Biological Chemistry, 276, 48937-48943. https://doi.org/10.1074/jbc.M107274200

[21] Hengst, L. (2004) A Second RING to Destroy p27Kip1. Nature Cell Biology, 6, 1153-1155. https://doi.org/10.1038/ncb1204-1153

[22] Soos, T.J., Kiyokawa, H., Yan, J.S., et al. (1996) Formation of p27-CDK Complexes during the Human Mitotic Cell Cycle. Cell Growth and Differentiation, 7, 135-146.

[23] Viglietto, G., Motti, M.L., Bruni, P., et al. (2002) Cytoplasmic Relocalization and Inhibition of the Cyclin-Dependent Kinase Inhibitor p27(Kip1) by PKB/Akt-Mediated Phosphorylation in Breast Cancer. Nature Medicine, 8, 1136-1144. https://doi.org/10.1038/nm762

[24] Shin, I., Yakes, F.M., Rojo, F., et al. (2002) PKB/Akt Mediates Cell-Cycle Progression by Phosphorylation of p27(Kip1) at Threonine 15 and Modulation of Its Cellular Localization. Nature Medicine, 8, 1145-1152. https://doi.org/10.1038/nm759

[25] Liang, J., Zubovitz, J., Petrocelli, T., et al. (2002) PKB/Akt Phosphorylates p27, Impairs Nuclear Import of p27 and Opposes p27-Mediated G1 Arrest. Nature Medicine, 8, 1153-1160. https://doi.org/10.1038/nm761

[26] Connor, M.K., Kotchetkov, R., Cariou, S., et al. (2003) CRM1/RAN-Mediated Nuclear Export of p27 Kip1 Involves a Nuclear Export Signal Please Cite and Links p27 Export and Proteolysis. Molecular Biology of the Cell, 14, 201-213. https://doi.org/10.1091/mbc.e02-06-0319 
[27] Ciarallo, S., Subramanian, V., Hung, W., Lee, J.H., Kotchetkov, R., Sandhu, C., et al. (2002) Altered p27Kip1 Phosphorylation, Localization, and Function in Human Epithelial Cells Resistant to Transforming Growth Factor $\beta$-Mediated G1 Arrest. Molecular Cell Biology, 22, 2993-3002.

https://doi.org/10.1128/MCB.22.9.2993-3002.2002

[28] Chu, I., Sun, J., Arnaout, A., et al. (2007) p27 Phosphorylation by Src Regulates Inhibition of Cyclin E-Cdk2. Cell, 128, 281-294.

https://doi.org/10.1016/j.cell.2006.11.049

[29] Kazi, A., Carie, A., Blaskovich, M.A., Bucher, C., Thai, V., Moulder, S., et al. (2009) Blockade of Protein Geranylgeranylation Inhibits Cdk2-Dependent p27Kip1 Phosphorylation on Thr187 and Accumulates p27Kip1 in the Nucleus: Implications for Breast Cancer Therapy. Molecular Cell Biology, 29, 2254-2263. https://doi.org/10.1128/MCB.01029-08

[30] Hsu, T.C., Nair, R., Tulsian, P., Camalier, C.E., Hegamyer, G.A., Young, M.R. and Colburn, N.H. (2001) Transformation Nonresponsive Cells Owe Their Resistance to Lack of p65/Nuclear Factor- $\kappa$ B Activation. Cancer Research, 61, 4160-4168. 